\title{
QTLs underlying natural variation in stele and xylem structures of rice root
}

\author{
Yusaku Uga*1), Kazutoshi Okuno ${ }^{1,2)}$ and Masahiro Yano ${ }^{1)}$ \\ 1) National Institute of Agrobiological Sciences, 2-1-2 Kannondai, Tsukuba, Ibaraki 305-8602, Japan \\ 2) Present address: University of Tsukuba, 1-1-1 Tennodai, Tsukuba, Ibaraki 305-8572, Japan
}

Rice cultivars show a wide range of variation in stele and xylem structures of the root as well as in root thickness (RTH). We identified quantitative trait loci (QTLs) for stele and xylem structures of the root by using $117 \mathrm{~F}_{3}$ lines from a cross between the lowland rice cultivar IR64 (thin roots) and the upland rice cultivar Kinandang Patong (thick roots). QTL analysis was performed using genotype data consisting of 197 DNA markers in $F_{2}$ plants and phenotype data of the $F_{3}$ plants. Stele transversal area (STA), total area and number of late metaxylem vessels (MXA and MXN) and RTH were measured in basal cross sections of nodal roots. A total of 10 QTLs, 2 for STA, 4 for MXA, 2 for MXN and 2 for RTH, were detected on chromosomes 1, 2, 3, 9 and 10. The Kinandang Patong allele at all QTLs showed a positive additive effect on each trait, except for one QTL for MXA on chromosome 10. The phenotypic variance explained by each QTL ranged from $8.7 \%$ to $23.9 \%$. A QTL for MXA on chromosome 9 showed the largest effect $(23.9 \%)$ on total phenotypic variance. Although one QTL for STA, detected on chromosome 2, was mapped near a QTL for RTH, the other QTLs for stele and xylem structures did not map to the same chromosomal regions as the QTLs for RTH. We conclude that stele and xylem structures might be controlled by several genetic factors different from the QTLs for RTH.

Key Words: Oryza sativa L., root anatomy, vascular system, root thickness, quantitative trait locus.

\section{Introduction}

In rice, root traits are key to drought avoidance because they can enable the plant to avoid water stress by absorbing water deposited in the deep soil layers (Yoshida and Hasegawa 1982). Root morphological traits such as thickness, maximum length, volume and distribution were suggested to contribute to drought avoidance in the field (Yoshida and Hasegawa 1982, Fukai and Cooper 1995). A wide range of genetic variation has been observed in such traits (reviewed by O'Toole and Bland 1987). In general, the roots of upland rice cultivars are morphologically thicker and penetrate more deeply than those of lowland cultivars. These traits are controlled by multiple genes (Ekanayake et al. 1985). Many analyses of quantitative trait loci (QTLs) of root morphological traits have been carried out using different mapping populations (reviewed by Price et al. 2002a). As a result, many QTLs for rice root thickness (RTH) have been genetically identified on all chromosomes (Champoux et al. 1995, Price and Tomos 1997, Yadav et al. 1997, Ali et al. 2000, Zheng et al. 2000, Zhang et al. 2001, Kamoshita et al. 2002a, 2002b, Price et al. 2002b, Venuprasad et al. 2002, Courtois et al. 2003). RTH has also been targeted for improvement of root morphology by marker-assisted selection (MAS) (Steele et al. 2006).

Communicated by H. Kato

Received July 30, 2007. Accepted October 20, 2007.

*Corresponding author (e-mail: yuga@affrc.go.jp)
The stele (vascular cylinder), which includes the xylem and phloem of the root, is very important for the absorption and translocation of water and nutrients. In particular, xylem elements in the root are directly associated with water transport from root to shoot. Axial conductance significantly affects the rate of water uptake from the soil by a crop (Richards and Passioura 1981, Hasegawa and Yoshida 1982). The size and number of xylem vessels influence their conductivity for water transport (Kondo et al. 2000). Widediameter xylem vessels have large axial conductance with respect to water flow, enhancing water uptake compared with that by thin-diameter vessels (Fukai and Cooper 1995).

Both root anatomy and root morphology have been well studied in rice (reviewed by Morita and Nemoto 1995). Rice cultivars exhibit broad variation in stele and late metaxylem vessel (MXVII) diameters or areas (Terashima et al. 1987, Kondo et al. 2000). Kondo et al. (2000) compared nodal root anatomy in 12 upland and lowland rice cultivars both in hydroponic culture and under field conditions. Traditional upland japonica cultivars had the largest stele and MXVII diameters under both conditions. We also observed stele and MXVII differences in 61 rice accessions, including both upland and lowland rice cultivars, grown under field conditions (unpublished data), and found that transversal areas of stele and MXVII in upland rice were larger than those in lowland rice. Although a wide range of variation among cultivars has been found, QTLs for root stele and xylem structures have not been reported previously, presumably because it is more laborious and time-consuming to measure 
these traits in a huge number of plants under a microscope than other root morphological traits.

The genetic relationship between QTLs for stele and xylem structures, and QTLs for RTH is not well known. Therefore, map information on QTLs for stele and xylem structures and on QTLs for RTH is required for genetic improvement of root traits associated with improved water uptake. In this study, to identify the genetic factors involving the stele and xylem structures and to clarify the relationship between them and RTH, we performed a QTL analysis of both types of traits using the $F_{3}$ population from a cross between the lowland cultivar IR64 (thin roots) and the upland cultivar Kinandang Patong (thick roots).

\section{Materials and Methods}

\section{Plant materials and cultivation}

An $\mathrm{F}_{3}$ population consisting of 117 lines derived from a cross between IR64 and Kinandang Patong was used for the QTL analysis in this study. IR64 is a modern lowland cultivar (indica) developed by the International Rice Research Institute in the Philippines and widely grown South and Southeast Asia. Kinandang Patong is a traditional upland cultivar (tropical japonica) that originated in the Philippines. Kondo et al. (2000) reported that diameters of root, stele, and MXVII of Kinandang Patong were clearly larger than those of IR64. Therefore, we used this mapping population to detect QTLs underlying the differences in stele and xylem structures found between lowland and upland rice.

Rice plants were grown under upland conditions for six weeks in summer 2005 at the National Institute of Agrobiological Sciences (NIAS) in Tsukuba, Japan. A randomized complete block design was adopted with two replicates. In each block, we raised a total of $42 \mathrm{~F}_{3}$ plants per line. Fourteen plants (seven in each block) for $F_{3}$, both parents, and $F_{1}$ were randomly selected for the measurement of root anatomical traits.

\section{Measurement of root anatomical traits}

We measured root stele and xylem structures of the main culm of the plant (Fig. 1). After fixing the main culm with roots in FAA solution (5\% formalin, 5\% acetic acid, $45 \%$ ethanol and $45 \% \mathrm{H}_{2} \mathrm{O}$ ), we randomly selected three of the lower nodal roots of the third node (counted from the highest rooting node) and cut them out according to the procedure of Terashima et al. (1987) (Fig. 1A). Terashima et al. (1987) reported that their method could properly evaluate variations of RTH and vascular system in different varieties, so it was adopted for use in this study. A cross section of a nodal root was then cut $3 \mathrm{~cm}$ from the base of the root with a plant microtome (MTH-1; Nippon Medical \& Chemical Instruments Co., Ltd., Osaka, Japan) and stained with toluidine blue. A digital image was taken of each cross section, and stele transversal area (STA), total transversal area and number of MXVII (MXA and MXN) and RTH were quantified under a digital microscope (VHX-200; Keyence Co., Ltd., Osaka, Japan) (Fig. 1B, 1C, 1D, 1E). To quantify the xylem structure, we focused on only MXVII, which exhibits the largest variation in xylem elements among rice cultivars (unpublished data). In general, 42 images $\left(14 \mathrm{~F}_{3}\right.$ plants $\times 3$ nodal roots) were used to calculate a mean value for each $\mathrm{F}_{3}$ line.

\section{Statistical analysis using phenotype data}

Heritability was estimated from the variances of the parent plants and the $F_{1}$ plants, variance among the $F_{3}$ lines and variance in the $\mathrm{F}_{3}$ line. Heritability was calculated as

$$
h^{2}=(3 A / 4+3 D / 32) /(3 A / 4+3 D / 32+E),
$$

where $A$ is the sum of squares of additive effects of all genetic factors related to the traits, $D$ is that of dominance effects of the genetic factors and $E$ is environmental variance.

\section{DNA marker analysis of the $F_{2}$ population}

A total of 197 DNA markers distributed over the 12 rice chromosomes were used for the QTL analysis: 111 simple sequence repeat (SSR) (Akagi et al. 1996, Temnykh et al.

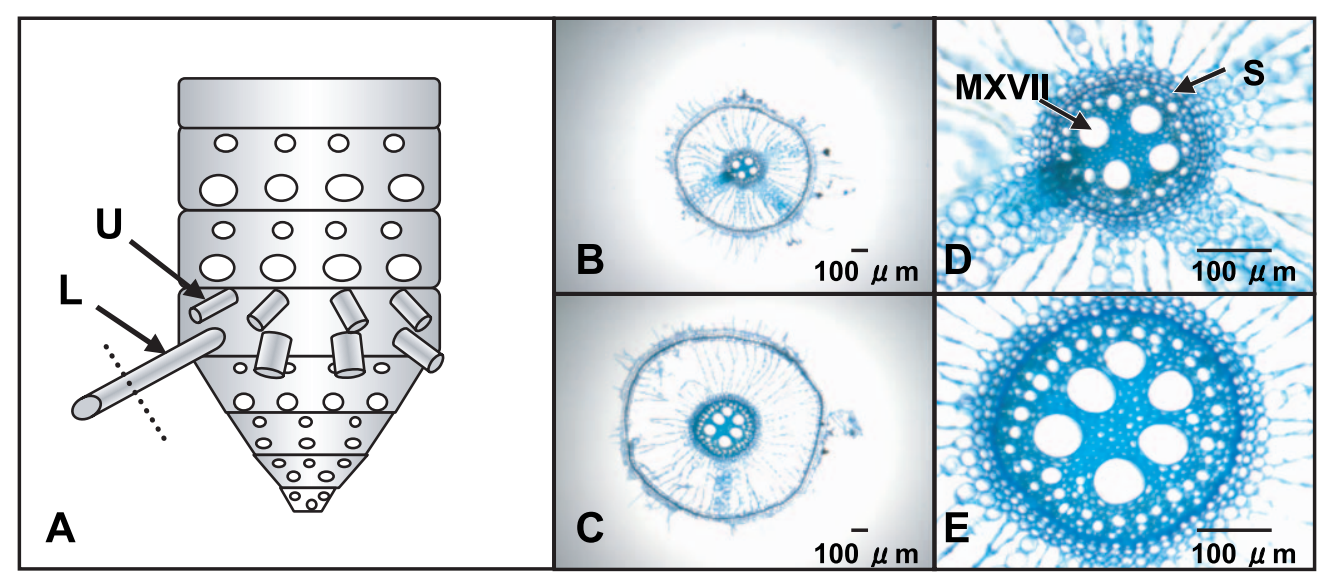

Fig. 1. Phenotypic differences in stele and xylem structures in rice root. (A) Scheme of nodal root. U, upper nodal root; L, lower nodal root. The dotted line indicates the site of the cross section. (B-E) Transverse root sections of rice plants stained with toluidine blue, showing the average value in each parent. S, stele; MXVII, late metaxylem vessel. (B) IR64; (C) Kinandang Patong; (D) IR64; (E) Kinandang Patong. 
2001), 43 sequence-tagged site (STS) (Wu et al. 2002) and 43 insertion-deletion (InDel) markers were used for genotyping the $\mathrm{F}_{2}$ plants. The InDel markers were constructed on the basis of information in the Rice DNA polymorphism database (Shen et al. 2004). The InDel markers used in this study are listed in the Appendix. Total DNA of plants was extracted from leaves by the CTAB method (Murray and Thompson 1980). PCR analysis was performed in a 5- $\mu$ l reaction mixture containing $1 \mu \mathrm{l}(20 \mathrm{ng}) \mathrm{DNA}, 0.5 \mu \mathrm{l} 10 \times \mathrm{PCR}$ buffer, $2 \mathrm{mM}$ dNTPs, $0.02 \mu \mathrm{l}$ (5 units) Ex Taq DNA polymerase (Takara Bio Inc., Otsu, Japan), $0.12 \mu \mathrm{l}$ of $20 \mathrm{pM}$ solutions of both primers and $2.86 \mu \mathrm{H}_{2} \mathrm{O}$. PCR was carried out in 35 cycles of $15 \mathrm{~s}$ of denaturation at $93^{\circ} \mathrm{C}, 30 \mathrm{~s}$ of annealing at $55^{\circ} \mathrm{C}$ for SSR markers and $60^{\circ} \mathrm{C}$ for STS and InDel markers and 2 min of extension at $72^{\circ} \mathrm{C}$. To detect polymorphism, the PCR products were electrophoresed in $3 \%$ agarose gels at $150 \mathrm{~V}$ for $90 \mathrm{~min}$.

\section{Construction of linkage map and QTL analysis}

Linkage maps were constructed from the genotype data with MAPMAKER/EXP 3.0 (Lander et al. 1987). The genetic distance was estimated by using the Kosambi map function (Kosambi 1944).

Putative QTLs were detected by using the composite interval mapping (CIM) function of QTL Cartographer 2.0
(Basten et al. 1994). The CIM threshold was based on the results of 1000 permutation tests at the $5 \%$ level of significance (Churchill and Doerge 1994). The additive and dominance effects and the phenotypic variance explained by each QTL $\left(R^{2}\right)$ were estimated at maximum LOD score. The total phenotypic variance explained by all of the detected QTLs was estimated by the multiple interval mapping (MIM) model of QTL Cartographer 2.0. To detect epistatic interactions between the detected QTLs, we performed two-way ANOVA using the genotype data of the marker nearest to each QTL.

\section{Results}

\section{Phenotypic variation of root anatomical traits}

Values of all traits in Kinandang Patong were significantly larger than those in IR64 (Fig. 1B, 1C, 1D, 1E and Fig. 2). In particular, MXA of Kinandang Patong (16661 $\left.\mu \mathrm{m}^{2}\right)$ was almost three times that of IR64 $\left(5942 \mu \mathrm{m}^{2}\right)$. The values of most traits of $F_{1}$ plants were intermediate between those of the parents, but their RTH values were similar to the Kinandang Patong value, suggesting that RTH of $\mathrm{F}_{1}$ plants could be attributed to dominance effects of the related genetic factor(s) more than to the other traits.

All traits in $\mathrm{F}_{3}$ lines showed normal distribution within the range of the parental values (Fig. 2). Positive phenotypic
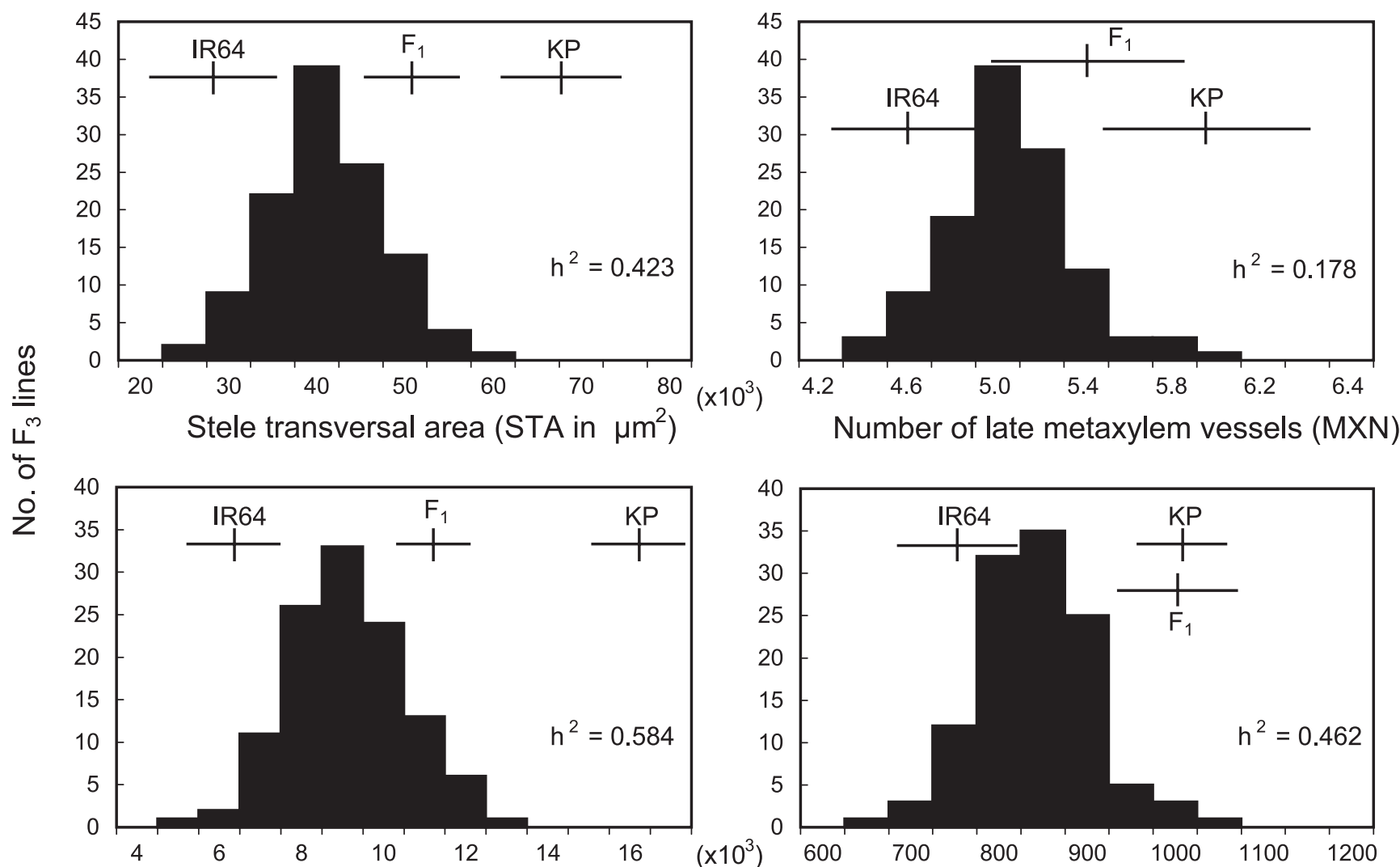

Total area of late metaxylem vessels (MXA in $\mu \mathrm{m}^{2}$ )

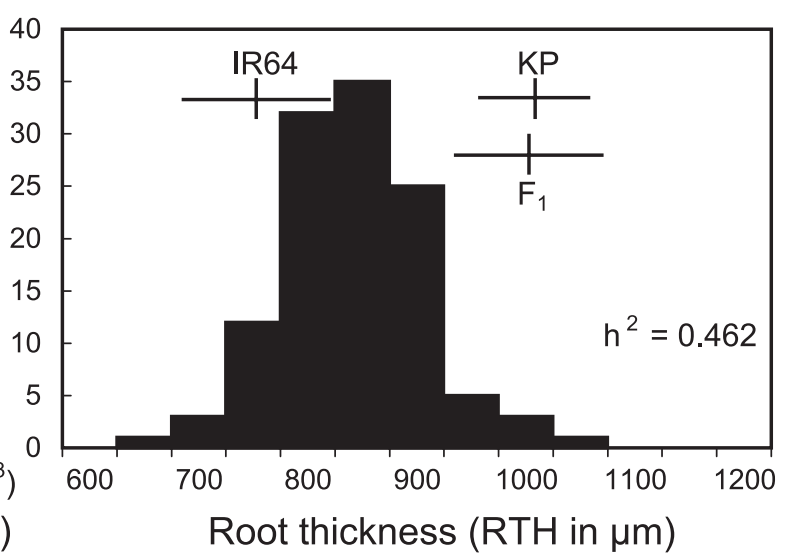

Fig. 2. Frequency distributions of four root anatomical traits in the $\mathrm{F}_{3}$ population derived from IR64 $\times$ Kinandang Patong (KP). Vertical and horizontal bars indicate the average value and standard deviation of each line, respectively. $h^{2}$ indicates heritability. 
correlations were observed between three stele and xylem structures (Table 1). In particular, a highly significant correlation was observed between MXA and STA $(r=0.923)$. The RTH and three stele and xylem structures also showed a positive correlation, ranging from 0.408 to 0.879 .

Heritability estimates of the four traits ranged from low (MXN, 0.178) to intermediate (MXA, 0.584) (Fig. 2).

\section{QTL analysis of root anatomical traits}

The $\mathrm{F}_{2}$ linkage map, composed of 197 markers, covered almost the whole rice genome. The total map distance was $1539.6 \mathrm{cM}$, and the average distance between markers was 8.2 $\mathrm{cM}$. We found eight QTLs for stele and xylem structures and two for RTH on this linkage map (Table 2 and Fig. 3). Twoway ANOVA revealed that no epistatic interaction occurred between the QTLs detected for each trait (data not shown).

Two QTLs for STA, qSTA-2 $\left(R^{2}=14.7 \%\right)$ and $q S T A-9$ $\left(R^{2}=16.2 \%\right)$, were detected on chromosomes 2 and 9 (Table 2 and Fig. 3). The additive effects of the Kinandang Patong alleles of the two QTLs were 3612 and $3434 \mu \mathrm{m}^{2}$, respectively, and their dominance effect values were 1123 and $-88 \mu \mathrm{m}^{2}$.

Four QTLs for MXA were detected on chromosomes 2, 9 and 10 (Table 2 and Fig. 3). Their $R^{2}$ values ranged from $8.7 \%$ to $23.9 \%$. Except for $q M X A-10$, the additive and dominance effects of the Kinandang Patong allele at all QTLs increased MXA, by from 657 to $947 \mu^{2}$ and from 48 to 300 $\mu \mathrm{m}^{2}$, respectively. By contrast, the Kinandang Patong allele at $q M X A-10$ decreased MXA.

Two QTLs for MXN, $q M X N-3$ and $q M X N-9$, were detected on chromosomes 3 and 9 (Table 2 and Fig. 3). Their $R^{2}$ values were $13.4 \%$ and $11.9 \%$, respectively. The additive effects of the Kinandang Patong alleles of the two QTLs were 0.14 and 0.15 , respectively, and the dominance effect
Table 1. Coefficients of correlation among four root anatomical traits in the $\mathrm{F}_{3}$ population derived from IR64 $\times$ Kinandang Patong

\begin{tabular}{llll}
\hline \hline & STA & MXA & MXN \\
\hline MXA & $0.9234^{* *}$ & & \\
MXN & $0.5597^{* *}$ & $0.6112^{* *}$ & \\
RTH & $0.8791^{* *}$ & $0.7625^{* *}$ & $0.4084^{* *}$ \\
\hline
\end{tabular}

STA, stele transversal area; MXA, total area of late metaxylem vessels; MXN, number of late metaxylem vessels; RTH, root thickness. ** $P<0.01$.

values were -0.04 and 0.01 .

Two QTLs for RTH, $q R T H-1$ and $q R T H-2$, were detected on chromosomes 1 and 2 (Table 2 and Fig. 3 ). The $R^{2}$ values of $q R T H-1$ and $q R T H-2$ were $21.0 \%$ and $19.3 \%$, respectively. The additive effects of the Kinandang Patong alleles of the two QTLs were 41.9 and $40.1 \mu \mathrm{m}$, respectively, and their dominance effects were 15.9 and $21.6 \mu \mathrm{m}$.

Among the 10 QTLs detected in this study, some QTLs for different traits were detected in particular chromosomal regions (Fig. 3). QTLs $q S T A-9$ and $q M X A-9$ overlapped near InDel marker ID07_14 on chromosome 9, and $q S T A-2$ and $q R T H-2$ both lay near SSR marker RM262 on chromosome 2. Other QTLs were each located in a different chromosomal region.

\section{Discussion}

Many researchers have analyzed QTLs for RTH in rice because it is an important trait associated with drought avoidance (reviewed by Price et al. 2002a). On the other hand, no genetic analysis of stele and xylem structures in root has been performed. It may be more important to focus

Table 2. Putative QTLs for four root anatomical traits detected in the $\mathrm{F}_{3}$ population derived from IR64 $\times$ Kinandang Patong

\begin{tabular}{|c|c|c|c|c|c|c|c|c|}
\hline Traits & QTL & Chr. & Nearest marker & $\mathrm{cM}^{a}$ & $\mathrm{LOD}^{b}$ & $A^{c}$ & $D^{d}$ & $R^{2 e}$ \\
\hline \multirow[t]{2}{*}{ STA } & $q S T A-2$ & 2 & RM262 & 0.0 & 7.0 & 3612 & 1123 & 14.7 \\
\hline & $q S T A-9$ & 9 & ID07_14 & 1.2 & 7.6 & 3434 & -88 & $\begin{array}{l}16.2 \\
34.3^{f}\end{array}$ \\
\hline \multirow[t]{4}{*}{ MXA } & $q M X A-2-1$ & 2 & OSR9A & 0.0 & 4.9 & 657 & 219 & 8.7 \\
\hline & $q M X A-2-2$ & 2 & S5302 & 3.0 & 6.8 & 796 & 300 & 16.3 \\
\hline & $q M X A-9$ & 9 & ID07_14 & 1.2 & 11.2 & 947 & 48 & 23.9 \\
\hline & $q M X A-10$ & 10 & ID12_14 & 0.0 & 5.0 & -673 & -121 & $\begin{array}{c}9.5 \\
52.3^{f}\end{array}$ \\
\hline \multirow[t]{2}{*}{ MXN } & $q M X M-3$ & 3 & RM168 & 1.5 & 5.0 & 0.14 & -0.04 & 13.4 \\
\hline & $q M X N-9$ & 9 & RM201 & 0.0 & 4.7 & 0.15 & 0.01 & $\begin{array}{l}11.9 \\
26.7^{f}\end{array}$ \\
\hline \multirow[t]{2}{*}{ RTH } & $q R T H-1$ & 1 & RM3810 & 3.2 & 7.6 & 41.9 & 15.9 & 21.0 \\
\hline & $q R T H-2$ & 2 & RM262 & 0.0 & 7.9 & 40.1 & 21.6 & $\begin{array}{l}19.3 \\
41.3^{f}\end{array}$ \\
\hline
\end{tabular}

\footnotetext{
${ }^{a}$ Genetic distance from the QTL LOD peak to the nearest marker.

${ }^{b}$ LOD thresholds for CIM of STA, MXA, MXN, and RTH were 4.0, 4.0, 3.8, and 3.9, respectively.

${ }^{c}$ Additive effect of the allele from Kinandang Patong compared with that from IR64.

${ }^{d}$ Dominance effect of the allele from Kinandang Patong compared with that from IR64.

${ }^{e}$ Percentage phenotypic variance explained by each QTL.

${ }^{f}$ Percentage phenotypic variance explained by multiple QTLs.
} 

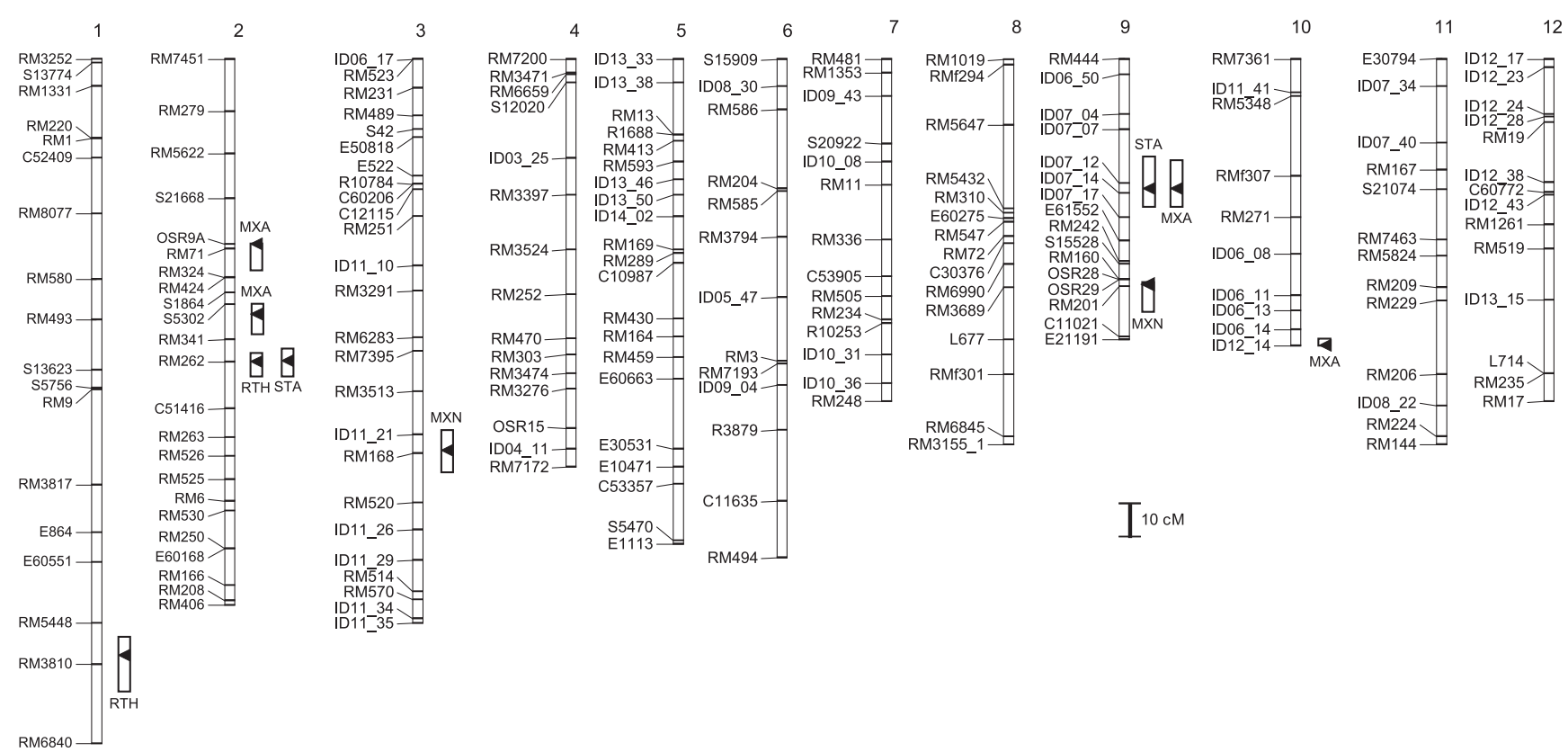

Fig.3. Chromosomal locations of QTLs for four root anatomical traits in rice. Chromosome numbers are indicated above each linkage map. Marker names are indicated on the left side of each linkage map. Triangles and white boxes on the right of each chromosome represent LOD peaks of putative QTLs and their one-LOD support intervals (Lynch and Walsh 1998), respectively.

on stele and xylem structures than on RTH from the viewpoint of water uptake from root to shoot. If the genes affecting the development of the stele and xylem are different from those affecting RTH, we should consider improving the stele and xylem structures independently from RTH. In this study, to clarify the genetic relationship between stele and xylem structures and RTH, we analyzed QTLs of stele and xylem structures in $F_{2}$ and $F_{3}$ populations. As a result, we successfully identified eight QTLs for stele and xylem structures. Total phenotypic variance of the detected QTLs, estimated by the MIM model, was almost equivalent to the heritability of each trait in the $\mathrm{F}_{3}$ lines. Therefore, we assumed that the major QTLs for stele and xylem structures could be found in this population. However, the accuracy of QTL detection might be limited in this population because we investigated only 14 plants in each $\mathrm{F}_{3}$ line, which are segregated generations. To reconfirm QTL detection, we should perform further analysis using advanced backcross progeny.

The eight QTLs for stele and xylem structures were detected on chromosomes 2, 3, 9 and 10. Among them, six were found on chromosomes 2 and 9, suggesting that both chromosomal regions play a key role in developing stele and xylem structures. On chromosome 2, $q M X A-2-1, q M X A-2-2$ and $q S T A-2$ were located in the interval between OSR9A and RM262 (about $35 \mathrm{cM}$ ), whereas $q S T A-9$ was found at ID07_14 on chromosome 9, where $q M X A-9$, which showed the largest $R^{2}$ (23.9\%) among all detected QTLs, was also identified. STA was highly correlated with MXA $(r=0.923)$. QTLs for several different traits showing a high level of phenotypic correlation are often detected in the same chromosomal regions (Paterson et al. 1991, Xiao et al. 1996). This QTL analysis demonstrated that the correlation between the
STA and MXA can be attributed either to a tight linkage of the QTLs for the traits or to pleiotropy of one QTL. Kawata et al. (1979) reported that the differentiation and maturation processes of each vascular element of a nodal root in rice show close morphological correlation, which may imply that one major QTL is involved in development of both STA and MXA. Further analysis, such as fine mapping and positional cloning of the QTLs $q S T A-9$ and $q M X A-9$, is needed to clarify the genetic relationship between STA and MXA during vascular system development. The two QTLs for MXN were detected in different chromosomal regions from those for STA and MXA, indicating that the number of MXVII and their total area are controlled by different genetic factors. However, MXN showed an intermediate level of correlation with MXA $(r=0.611)$. When QTLs with large effects segregate simultaneously in primary populations such as the $F_{2}$ generation, it is difficult to detect QTLs with small effects (Yano and Sasaki 1997). Therefore, to reveal the genetic relationship between the MXN and MXA, fine mapping of the QTLs for both MXN and MXA using advanced backcross progeny will be necessary.

To understand the genetic relationships between stele and xylem structures and RTH, we compared the chromosomal locations of the QTLs for these traits. In the population used in this study, although we observed a high level of phenotypic correlation between stele and xylem structures and RTH (Table 1), QTLs for stele and xylem structures were not shared with the two QTLs for RTH, except for qSTA-2 (Fig. 3). This result suggests that the genetic factors for stele and xylem structures and RTH are different. The QTL $q R T H-1$ may play an important role in the development of RTH in rice because several QTLs for RTH have been 
found near it in other studies (Zheng et al. 2000, Kamoshita et al. 2002a, Price et al. 2002b, Courtois et al. 2003), while only one QTL for RTH was reported near the region of the QTL $q R T H-2$ (Venuprasad et al. 2002), according to marker positions based on physical map information published by the International Rice Genome Sequencing Project (2005). However, QTLs for stele and xylem structures were not identified in this region. On the other hand, comparison of the chromosomal locations of QTLs for RTH detected in other studies showed that $q S T A-9$ and $q M X A-9$ are located near regions of two RTH QTLs with small effects, detected between markers Amy3ABC and RZ228 in an IR64/Azucena population (Zheng et al. 2000) and at marker G385 in a Bala/Azucena population (Price et al. 2002b). Furthermore, in this study, although it was not statistically significant, a LOD peak (LOD score $=2.9$ ) for RTH was observed in the interval defined by markers E61552 and RM242 on chromosome 9 near from the region detected $q S T A-9$ and $q M X A-9$ (data not shown). Therefore, the possibility that both stele and xylem structures and RTH are controlled by the same QTLs cannot be ruled out. To obtain a clear resolution of this problem, further analysis is required, such as fine mapping using advanced backcross progeny.

$\mathrm{RTH}$ is one of the most reproducible parameters among root traits, which are favorably associated with pre-heading drought avoidance (Champoux et al. 1995). RTH is also relatively easy to measure, even in the field (Yadav et al. 1997). By contrast, stele and xylem structures are laborious and time-consuming to evaluate. Therefore, many researchers interested in root morphology have focused on QTLs for $\mathrm{RTH}$ rather than on those for stele and xylem structures. However, our results suggest that natural variations in stele and xylem structures are generated by the combination of several alleles at QTLs different from those for RTH. This finding implies that we need to consider not only RTH but also stele and xylem structures to improve the water uptake capacity of roots. However, selection of stele and xylem structures in a large number of individuals by phenotyping is very difficult. The map information of QTLs for stele and xylem structures obtained in this study opens the way for improvement of stele and xylem structures through MAS and clarifies the genetic mechanism of their natural variation.

\section{Acknowledgments}

We thank Drs. S. Morita, J. Abe and M. Kondo for their technical advice regarding the anatomical measurement of rice roots. We also thank Dr. T. Hayashi for statistical advice regarding the genetic analysis and Dr. S. Yamanaka and the staff of the technical support section of the NIAS for field management and support during sampling. We are grateful to Dr. S. Fukuoka for constructing primers of InDel markers. This work was supported by a grant from the Ministry of Agriculture, Forestry and Fisheries of Japan (Agri-Genome Project QT-2001).

\section{Literature Cited}

Akagi,H., Y.Yokozeki, A.Inagaki and T.Fujimura (1996) Microsatellite DNA markers for rice chromosomes. Theor. Appl. Genet. 93: 1071-1077.

Ali,M.L., M.S.Pathan, J.Zhang, G.Bai, S.Sarkarung and H.T.Nguyen (2000) Mapping QTLs for root traits in a recombinant inbred population from two indica ecotypes in rice. Theor. Appl. Genet. 101: 756-766.

Basten,C.J., B.S.Weir and Z.B.Zeng (1994) Zmap-a QTL cartographer. Computing Strategies and Softwear. Proc. 5th Congr. on Genetics Applied. to Livestoc. Kinandang Patongroduction, Guelph, Ontario.

Champoux, M.C., G.Wang, S. Sarkarung, D.J.Mackill, J.C.O'Toole, N.Huang and S.R.McCouch (1995) Locating genes associated with root morphology and drought avoidance in rice via linkage to molecular markers. Theor. Appl. Genet. 90: 969-981.

Churchill,G.A. and R.W.Doerge (1994) Empirical threshold values for quantitative trait mapping. Genetics 138: 963-971.

Courtois,B., L.Shen, W.Petalcorin, S.Carandang, R.Mauleon and Z.Li (2003) Locating QTLs controlling constitutive root traits in the rice population IAC $165 \times$ Co39. Euphytica 134: 335-345.

Ekanayake,I.J., J.C.O'Toole, D.P.Garrity and T.M.Masajo (1985) Inheritance of root characters and their relations to drought resistance in rice. Crop Sci. 25: 927-933.

Fukai,S. and M.Cooper (1995) Development of drought-resistant cultivars using physio-morphological traits in rice. Field Crops Res. 40: 67-86.

Hasegawa,S. and S. Yoshida (1982) Water uptake by dryland rice root system during soil drying cycle. Soil Sci. Plant Nutr. 28: 191204.

International Rice Genome Sequencing Project (2005) The map-based sequence of the rice genome. Nature 436: 793-800.

Kamoshita,A., J.Zhang, J.Siopongco, S.Sarkarung, H.T.Nguyen and L.J.Wade (2002a) Effects of phenotyping environment on identification of quantitative trait loci for rice root morphology under anaerobic condition. Crop Sci. 42: 255-265.

Kamoshita,A., L.J.Wade, M.L.Ali, M.S.Pathan, J.Zhang, S.Sarkarung and H.T.Nguyen (2002b) Mapping QTLs for root morphology of a rice population adapted to rained lowland conditions. Theor. Appl. Genet. 104: 880-893.

Kawata, S., S.Morita and K.Yamazaki (1979) On the numbers of vessels and sieve tubes in crown roots of rice plants. Jpn. J. Crop Sci. 48: 502-509 (in Japanese with English summary).

Kondo,M., A.Aguilar, J.Abe and S.Morita (2000) Anatomy of nodal roots in tropical upland and lowland rice varieties. Plant Prod. Sci. 3: 437-445.

Kosambi,D.D. (1944) The estimation of map distance from recombination values. Ann. Eugenics 12: 172-175.

Lander, E.S., P. Green, J. Abrahamson, A. Barlow， M.J. Daley, S.E.Lincoln and L.Newburg (1987) MAPMAKER: an interactive computer package for constructing primary genetic linkage maps of experimental and natural populations. Genomics 1: 174-181.

Lynch,M. and B.Walsh (1998) Genetics and analysis of quantitative traits. Sinauer Associates, Sunderland, MA. p. 448-450.

Morita, S. and K. Nemoto (1995) Morphology and anatomy of rice roots with special reference to coordination in organo- and histogenesis. In "Structure and function of roots" Baluška,F. et al. (ed.), Kluwer Academic Publishers, Netherlands. p. 75-86.

Murray,M.G. and W.F.Thompson (1980) Rapid isolation of high 
molecular weight plant DNA. Nucleic Acids Res. 8: 4321-4325.

O'Toole,J.C. and W.L.Bland (1987) Genotypic variation in crop plant root systems. Adv. Agron. 41: 91-143.

Paterson,A.H., S.Damon, J.D.Hewit, D.Zamir, H.D. Rabinowitch, S.E.Lincoln, E.S.Lander and S.D.Tanksley (1991) Mendelian factors underlying quantitative traits in tomato: comparison across species, generations, and environments. Genetics 127: 181-197.

Price,A.H. and A.D.Tomos (1997) Genetic dissection of root growth in rice (Oryza sativa L.). II: mapping quantitative trait loci using molecular markers. Theor. Appl. Genet. 95: 143-152.

Price,A.H., J.E.Cairns, P.Horton, H.G.Jones and H.Griffiths (2002a) Linking drought-resistance mechanisms to drought avoidance in upland rice using a QTL approach: progress and new opportunities to integrate stomatal and mesophyll responses. J. Exp. Bot. 53: 989-1004.

Price, A.H., K.A.Steele, B.J.Moore and R.G.W.Jones (2002b) Upland rice grown in soil-filled chambers and exposed to contrasting water-deficit regimes II. Mapping quantitative trait loci for root morphology and distribution. Field Crops Res. 76: 25-43.

Richards,R.A. and J.B.Passioura (1981) Seminal root morphology and water use if wheat. I. Environmental effects. Crop Sci. 21: 249-252.

Shen, Y.-J., H.Jiang, J.-P.Jin, Z.-B.Zhang, B.Xi, Y.-Y.He, G.Wang, C.Wang, L.Qian, X.Li, Q.-B.Yu, H.-J.Liu, D.-H.Chen, J.-H.Gao, H.Huang, T.-L.Shi and Z.-N.Yang (2004) Development of genome-wide DNA polymorphism database for map-based cloning of rice genes. Plant Physiol. 135: 1198-1205.

Steele, K.A., A.H.Price, H.E. Shashidhar and J.R.Witcombe (2006) Marker-assisted selection to introgress rice QTLs controlling root traits into an Indian upland rice variety. Theor. Appl. Genet. 112: 208-221.

Temnykh,S., G.DeClerck, A.Lukashova, L.Lipovich, S.Cartinhour and S.McCouch (2001) Computational and experimental analysis of microsatellites in rice (Oryza sativa L.): frequency, length variation, transposon associations, and genetic marker potential. Genome Res. 11: 1441-1452.
Terashima,K., H.Hiraoka and I.Nishiyama (1987) Varietal difference in the root of rice plant I. Varietal difference in the morphology of crown root. Jpn. J. Crop Sci. 56: 521-529 (in Japanese with English summary).

Venuprasad,R., H.E. Shashidhar, S.Hittalmani and G.S.Hemamalini (2002) Tagging quantitative trait loci associated with grain yield and root morphological traits in rice (Oryza sativa L.) under contrasting moisture regimes. Euphytica 128: 293-300.

Wu, J., T. Maehara, T. Shimokawa, S. Yamamoto, C. Harada, Y.Takazaki, N.Ono, Y.Mukai, K.Koike, J.Yazaki, F.Fujii, A.Shomura, T.Ando, I.Kono, K. Waki, K. Yamamoto, M.Yano, T.Matsumoto and T.Sasaki (2002) A comprehensive rice transcript map containing 6591 EST sites. Plant Cell 14: 525-535.

Xiao,J., J.Li, L.Yuan and S.D.Tanksley (1996) Identification of QTLs affecting traits of agronomic importance in a recombinant inbred population derived from a subspecific rice cross. Theor. Appl. Genet. 92: 230-244.

Yadav, R., B.Courtois, N.Huang and G.McLaren (1997) Mapping genes controlling root morphology and root distribution in a doubled-haploid population of rice. Theor. Appl. Genet. 95: 619-632.

Yano,M. and T.Sasaki (1997) Genetic and molecular dissection of quantitative traits in rice. Plant Mol. Biol. 35:145-153.

Yoshida,S. and S.Hasegawa (1982) The rice root system: its development and function. In "Drought resistance in crops with emphasis on rice" International Rice Research Institute, Los Baños, Laguna, Philippines. p. 97-114.

Zhang,J., H.G.Zheng, A.Aarti, G.Pantuwan, T.T.Nguyen, J.N.Tripathy, A.K. Sarial, S. Robin, R.C.Babu, B.D.Nguyen, S. Sarkarung, A.Blum and H.T.Nguyen (2001) Locating genomic regions associated with components of drought resistance in rice: comparative mapping within and across species. Theor. Appl. Genet. 103: 19-29.

Zheng,H., R.C.Babu, M.S.Pathan, L.Ali, N.Huang, B.Courtois and H.T.Nguyen (2000) Quantitative trait loci for root-penetration ability and root thickness in rice: comparison of genetic backgrounds. Genome 43: 53-61. 
Appendix. List of $43 \mathrm{InDel}$ markers

\begin{tabular}{|c|c|c|c|c|c|}
\hline Locus & Chromosome & $\begin{array}{l}\text { PCR product in } \\
\text { Nipponbare (bp) }\end{array}$ & Forward primer & Reverse primer & Anealing temp. \\
\hline ID06_17 & 3 & 216 & ttcgattcgacggtgtatca & tccattaatcgggaactcca & 60 \\
\hline ID11_10 & 3 & 160 & gtgggtccagtggcaaatag & ggtttctagcccaacattgc & 60 \\
\hline ID11_21 & 3 & 165 & gggtcc acatgtcattctc & cctctgattcgtctgcacagt & 60 \\
\hline ID11_26 & 3 & 239 & gaggcaaagctggatggtta & cccatggctgcacttatttt & 60 \\
\hline ID11_29 & 3 & 243 & tgaatcccccatagaagtgc & cccatgtgatgttgatgtgg & 60 \\
\hline ID11_34 & 3 & 173 & tgtgttgtgtggaagggaaa & tgcaagcaaccaaacacttc & 60 \\
\hline ID11_35 & 3 & 203 & cetttcttcetcctcgatcc & ggagagcagctggtgaagat & 60 \\
\hline ID03_25 & 4 & 187 & tttacacggaacttaggtcaga & tctcgcaatttacactccat & 60 \\
\hline ID04_11 & 4 & 154 & gccgatgagaatttggtcc & gttcgtaaggttcgggtgaag & 60 \\
\hline ID13_33 & 5 & 169 & gctgttggactcccgagata & tccgccetctcggtattctt & 60 \\
\hline ID13_38 & 5 & 225 & gagctgatgggatcggttttt & tagtgctcgtactgecgacctt & 60 \\
\hline ID13_46 & 5 & 152 & tgttcctctccttcctcagca & ttgctagggattgcctggag & 60 \\
\hline ID13_50 & 5 & 155 & gatgcgcgttgtcagagatg & tgtctcctctgcatgcgact & 60 \\
\hline ID14_02 & 5 & 164 & tgcaccttgtcaagcgtctc & ttttgtactgcaagggcgttag & 60 \\
\hline ID08_30 & 6 & 189 & agatgcaagcgggtatgaac & gaagcacagtcacaggacca & 60 \\
\hline ID05_47 & 6 & 170 & tcgttgagccetcatcgtct & cacccgatccagagtcttgtt & 60 \\
\hline ID09_04 & 6 & 197 & cgaaagggaaggagaaagc & agttcgtcgcacetcettt & 60 \\
\hline ID09_43 & 7 & 248 & cacccaaacacaagaaagca & attggtaattcccacgctca & 60 \\
\hline ID10_08 & 7 & 236 & ttgcctgctggctagtgaat & cgttcgcataccetatgacc & 60 \\
\hline ID $10 \_31$ & 7 & 184 & tcaccaatatgcaaccgaga & ttagcattgctttcggacct & 60 \\
\hline ID10_36 & 7 & 192 & ggtcgcctcaacttcaagag & gacaccaagcatcaactcca & 60 \\
\hline ID06_50 & 9 & 196 & cccacctaccaatcattca & gtgttggaagaggggagatg & 60 \\
\hline ID07_04 & 9 & 205 & cgtgctaaacctggtgtaaag & ggcatagtcatgcaccctct & 60 \\
\hline ID07_07 & 9 & 224 & ggccatgaaaggtggataac & atcggagttgtgaagcaacc & 60 \\
\hline ID07_12 & 9 & 219 & ttgttgagcaatgagcgaac & ggggctctcatttcaacctt & 60 \\
\hline ID07_14 & 9 & 174 & ccatgatcaaaccacacagc & tgtcagggcaccatgactta & 60 \\
\hline ID07_17 & 9 & 192 & aaaggcagagggatctaggc & cttggcatctatcaagggttg & 60 \\
\hline ID11_41 & 10 & 184 & tggaggaattggaggatgag & tggatccggtattgaaggag & 60 \\
\hline ID06_08 & 10 & 172 & accctcgcgaatttttaagc & tgtcagtgctcggttttgtc & 60 \\
\hline ID06_11 & 10 & 222 & agggacagggatggagaagt & ctcacaagtcgatgcacaca & 60 \\
\hline ID06_13 & 10 & 228 & cgtacgctctaccacgttga & ggatggttttggagttttgg & 60 \\
\hline ID06_14 & 10 & 247 & cacgggtgaacgtaaatcct & cettctcetttgectttcatc & 60 \\
\hline ID12_14 & 10 & 176 & ccatggagcaagaagatggt & ggcacgtagggtgatgagat & 60 \\
\hline ID07_34 & 11 & 216 & accacacagaaatcccaagc & acaactccaggagagccaga & 60 \\
\hline ID07_40 & 11 & 200 & cagcagcagcagatcaacag & gacaacaaccctcatgaca & 60 \\
\hline ID08_22 & 11 & 205 & atgtgtatgccgtgcatgtt & gggccaaattcaatcacaac & 60 \\
\hline ID12_17 & 12 & 172 & accgtagcgttagcatggac & actacgagaatgcggtgett & 60 \\
\hline ID12_23 & 12 & 196 & tggcttatggaagatgcaca & acgttttgctccttttcgag & 60 \\
\hline ID12_24 & 12 & 212 & ggcagaaagaataacagaccac & gaggcattttgtcaggcaat & 60 \\
\hline ID12_28 & 12 & 250 & cagctgcaacacggacttca & cgagtaccaacaaggcagca & 60 \\
\hline ID12_38 & 12 & 187 & ccetctttgecetcetgatt & tggtgttcctgcactggttg & 60 \\
\hline ID12_43 & 12 & 243 & ctcggtatagctacgttcaaatcg & cgagtgacaatcggacaattacc & 60 \\
\hline ID13_15 & 12 & 216 & tttcacgggtgcacagagatt & cgtggatctttcccatgctt & 60 \\
\hline
\end{tabular}

